\title{
INTERAÇÕES MEDICAMENTOSAS ASSOCIADAS À FARMACOLOGIA DA COVID-19 EM UM HOSPITAL PÚBLICO DO SERTÃO DE PERNAMBUCO
}

\author{
Maria Clara Inácio de Sá1*; Jorge Ederson Gonçalves Santana² \\ ${ }^{1}$ Discente do curso de Farmácia, Centro de Ensino Superior do Vale do São Francisco (CESVASF), \\ Belém do São Francisco, Pernambuco. \\ ${ }^{2}$ Coordenador e docente do Curso de Farmácia, Centro de Ensino Superior do Vale do São \\ Francisco, (CESVASF), Belém do São Francisco, Pernambuco.
}

DOI: 10.47094/IICNNESP.2021/87

\begin{abstract}
RESUMO
Introdução. Após um ano de pandemia, apenas o antiviral remdesivir foi aprovado no enfretamento hospitalar ao COVID-19, porém, a farmacoterapia desta infecção, demanda de diversos fármacos para manter a estabilidade respiratória do paciente e os seus sinais vitais. Metodologia: $\mathrm{O}$ trabalho foi desenvolvido a partir de uma análise das prescrições de enfrentamento ao COVID-19 no Hospital Regional Inácio de Sá, sendo selecionadas 33 prescrições de diferentes setores de internamento. A verificação das interações ocorreu a partir do site Medscape, sendo classificadas de acordo com o risco ao paciente. Resultado: Um total de 97\% das prescrições apresentou interações entre as drogas, sendo os fármacos azitromicina, enoxaparina, dexametasona e ceftriaxona mais frequentes. Apenas as prescrições pediátricas não apresentaram interações, por não utilizarem de múltiplas drogas na terapia. Considerações Finais: Foram identificadas diversas interações entre as drogas, sendo necessários estudos clínicos para verificar as reações adversas provenientes destas interações.

PALAVRAS-CHAVE: Sars-CoV-2; Pandemia; Farmacoterapia.

ÁREA TEMÁTICA: Condições sociais e de saúde.

\section{INTRODUÇÃO}

O SARS-CoV-2, vírus causador da COVID-19, foi identificado a partir de surtos em Hubei, província Chinesa, sendo um novo tipo de coronavírus com características de elevada transmissibilidade e rápida propagação, logo se espalhando por todo o planeta, levando a Organização Mundial da Saúde (OMS) a declarar, em março de 2020, Estado de Pandemia. Atualmente, o vírus já infectou uma estimativa de mais de 135 milhões de pessoas em todo o mundo. O primeiro caso do novo coronavírus no Brasil foi confirmado em fevereiro de 2020, e atualmente o país já conta com 13 milhões de infectados e 362 mil óbitos, sendo um local de alto risco de infecção. O quadro brasileiro agravou ainda mais a partir de março de 2021, com altas taxas de infecção e mortalidade (média de cerca de 3000 óbitos/dia) (OMS, 2021).
\end{abstract}


Desde o surgimento da COVID-19, muitos estudos em busca de medicamentos e vacinas foram desenvolvidos, sendo destaque o surgimento de mais de 10 tipos de vacinas consideradas eficazes e seguras. Em contrapartida, dos diversos fármacos testados na redução da carga viral, o único que se mostrou mais eficaz foi o remdesivir. Esse fármaco foi o primeiro antiviral recomendado para tratamento da COVID-19 nos Estados Unidos da América, Brasil e outros países do mundo. No entanto, trata-se de um fármaco para uso exclusivamente hospitalar em pacientes que não estejam entubados. Além disso, o medicamento ainda não está sendo produzido no Brasil e possui um elevado custo (um tratamento de 10 dias pode custar 18 mil reais) (OMS, 2021).

Diante disso, a recomendação ainda consiste em medidas de contenção e prevenção ao novo coronavírus, No caso de pacientes que se infectam com o Sars-CoV-2, recomenda-se o isolamento e acompanhamento dos órgãos públicos de saúde e vigilância epidemiológica. Na evolução do quadro e internação, os pacientes são tratados com medicamentos que reduzem os sintomas da COVID-19, tais como: antivirais, antimicrobianos, antiinflamatórios (AINEs e glicocorticóides), imunoduladores, dentre outros. Esses medicamentos visam manter a estabilidade respiratória do paciente, os seus sinais vitais e auxiliam o sistema imunológico a combater a infecção (ZHANG e LIU, 2020; DUARTE, et al, 2020).

O tratamento da COVID-19 em âmbito hospitalar baseia-se no recurso da farmacoterapêutica da polifarmácia, levando ao risco do surgimento de interações medicamentosas que podem ser consideradas um empecilho na eficácia da terapêutica. Levando em consideração essa característica, a pesquisa teve como objetivo a busca ativa por interações medicamentosas, através de uma análise das prescrições médicas de pacientes internado na ALA de tratamento da COVID-19 de um hospital da Rede Pública de Saúde do Estado de Pernambuco.

\section{METODOLOGIA}

A coleta dos dados ocorreu a partir de uma análise das prescrições médicas para tratamento ao COVID-19 no Hospital Regional Inácio de Sá, localizado na cidade de Salgueiro, no Sertão de Pernambuco, sendo este estudo caracterizado como quantitativo e descritivo. Os pacientes estavam internados na ala Covid-19 do hospital, desde a Emergência Respiratória até a Unidade de Terapia Intensiva (UTI). Foram selecionadas 33 prescrições entre os meses de outubro e novembro de 2020, levando em consideração apenas a terapia farmacológica e os medicamentos utilizados, sendo excluídos os dados pessoais dos pacientes. As interações medicamentosas foram consultadas através do site de busca "Medscape" e classificadas de acordo com os riscos, organizando em tabelas de acordo com sua frequência. Além disso, verificou-se na literatura científica a veracidade destas interações e o as consequências para o paciente. 


\section{RESULTADOS E DISCUSSÃO}

Entre as prescrições, 97\% apresentaram interações medicamentosas, sendo observada uma polifarmárcia na terapia. As prescrições que não apresentaram interações medicamentosas eram provenientes do setor pediátrico, onde os pacientes não faziam uso de múltiplos fármacos. Em algumas terapias observou-se um elevado índice de interações medicamentosas, apresentando entre vinte e seis e dezoito interações por prescrição. As interações medicamentosas são classificadas de acordo com o seu risco a saúde do paciente, ou seja, alto risco, risco moderado e baixo risco. O gráfico abaixo aborda a frequência destas interações sendo classificadas de acordo com o risco ao paciente.

Tabela 1. Frequência de interações por prescrição e os riscos ao paciente.

\begin{tabular}{|c|c|c|c|}
\hline Interação Medicamentosas & Frequência & Risco & Plataforma de Busca \\
\hline Azitromicina + Enoxaparina & 12 & Alto risco & Medscape \\
\hline Ceftriaxona + Enoxaparina & 13 & Alto risco & Medscape \\
\hline Dexametasona + Sinvastatina & 3 & Alto Risco & Medscape \\
\hline Dexametasona+ Enoxaparina & 17 & Moderado & Medscape \\
\hline Enoxaparina + Captopril & 6 & Moderado & Medscape \\
\hline Enoxaparina + Losartana & 8 & Moderado & Medscape \\
\hline Dexametasona + Aminofilina & 5 & Moderado & Medscape \\
\hline Dexametasona + Omeprazol & 16 & Baixo & Medscape \\
\hline Ceftriaxona + Furosemida & 7 & Baixo & Medscape \\
\hline Omeprazol + Aminofilina & 5 & Baixo & Baixo \\
\hline Dexametasona + Furosemida & 4 & & \\
\hline
\end{tabular}

Fonte: SÁ \& SANTANA (2020)

Os fármacos que apresentaram maior frequência de interações nas prescrições foram a azitromicina, enoxaparina, dexametasona e a ceftriaxona. Além disso, observou-se interação entre drogas de tratamento a patologias subjacentes do paciente, como o captopril, losartana, furosemida, ambas com utilização na terapia da Hipertensão Arterial Sistêmica (HAS), e a sinvastatina, utilizada para redução das dislipidemias. Outros fármacos identificados foram o omeprazol, essencial a proteção gástrica em casos de utilização de múltiplas drogas, e a aminofilina, utilizada como broncodilatador na terapia da COVID-19.

A terapia baseada em múltiplos fármacos é um fator para a ocorrência de interações medicamentosas, sendo proporcional à quantidade de fármacos empregados. Os pacientes internados em UTI's, utilizam-se da polifarmácia, aumentando o risco de interação medicamentosas, e consequentemente, reações adversas aos medicamentos (RAM's) (CEDRAZ e SANTOS, 2014).

Um estudo desenvolvido por Melo e Cols. (2021) constatou que na terapia da COVID-19, interações/as RAM's são mais comuns quando associa-se fármacos das classes de antimicrobianos aminoquinolonas e macrolídeos e agentes antitrombóticos, destacando-se hidroxicloroquina, azitromicina, ceftriaxona e enoxaparina. 
A utilização de anticoagulantes, antiagregantes plaquetários e estatinas são frequentemente correlacionadas aos casos de interações. Vários estudos demonstram a relação da enoxaparina com diversos fármacos como aspirina, clopidogrel e varfarina sódica, levando ao surgimento de hematomas, epistaxe e hematúria (SOBRINHO, et al, 2012). Yunes e cols. (2011) realizaram uma análise da farmacoterapia proveniente da UTI, relatando a ocorrência de interação medicamentosa entre a enoxaparina e a ceftriaxona, concluindo que os pacientes internados ficam vulneráveis às interações medicamentosas devido a polifármacia neste ambiente.

Estudos clínicos demonstram que a utilização da azitromicina adjunto as estatinas, exemplo a sinvastatina, podem aumentar o risco de rabdomiólise. Além disso, a utilização do antimicrobiano com fármacos que promovem o aumento do intervalo QT devem ser observados e monitorados (MCMULLAN e MOSTAGHIM, 2015). A azitromicina é responsável por aumentar a exposição de fármacos cardiotóxicos, estando também relacionada com eventos cardíacos graves e por cadiotoxicidade, devendo ser utilizada com cautela em pacientes infectados pelo COVID-19 (MACHIELS, e cols. 2020). Ainda não há estudos que monitorem a ocorrência de reações adversas e efeitos colaterais provenientes da polifarmácia na terapia do COVID-19, portanto, a farmacoterapia deve utilizar do mecanismo de monitoração e avaliar os riscos e benefícios ao paciente, ajustando o tratamento sempre que necessário.

Um estudo farmacocinético conduzido por Reddy et al. (2020) demonstrou que não há necessidade de uma mudança na dosagem da farmacoterapia baseada na idade ou raça, porém o paciente infectado pelo SARS-CoV-2 ao apresentar disfunções hepáticas sofre de um aumento à exposição de determinadas drogas, como a azitromicina, devendo ser utilizada com cautela e frequentemente monitorada.

\section{CONSIDERAÇÕES FINAIS}

A terapia ao COVID-19 demanda da utilização de múltiplos fármacos, sendo identificadas diversas interações entre as drogas, as quais possuem um risco de agravamento do quadro clínico do paciente. Fármacos como a enoxaparina, dexametasona, ceftriaxona e azitromicina demonstraram frequência relevante nas prescrições, com índices consideráveis de interação, sendo necessário um monitoramento do paciente. Além disso, estudos clínicos sobre as reações adversas provenientes de interações entre as drogas na terapia ao COVID-19 são bastante escassos, devendo ser objetivo de estudo das futuras pesquisas.

\section{REFERENCIAS}

CEDRAZ, K. N.; SANTOS, M. C. J. Identificação e caracterização de interações medicamentosas em prescrições médicas da unidade de terapia intensiva de um hospital público da cidade de Feira de Santana, BA. Rev Soc Bras Clin Med, v. 12, n. 2, p. 124-30, 2014.

DUARTE, D. B.; COELhO, L. J.; NASCIMENTO, G. N. L. Atualidades da farmacoterapia da 
COVID-19. Desafios-Revista Interdisciplinar Da Universidade Federal Do Tocantins, v. 7, n. Especial-3, p. 81-89, 2020.

MCMUllan, B. J.; MOSTAGHIM, M. Prescribing azithromycin. Australian prescriber, v. 38, n. 3, p. $87,2015$.

MELO, J. R. R., et al. Reações adversas a medicamentos em pacientes com COVID-19 no Brasil: análise das notificações espontâneas do sistema de farmacovigilância brasileiro. Cadernos de Saúde Pública, v. 37, p. e00245820, 2021.

MACHIELS, J. D. et al. Reply to Gautret et al: hydroxychloroquine sulfate and azithromycin for COVID-19: what is the evidence and what are the risks?. International journal of antimicrobial agents, p. 106056, 2020.

OMS, Organização Mundial da Saúde. Folha informativa COVID-19 - Escritório da OPAS e da OMS no Brasil. Organização Panamericana de Saúde. 2020. Acesso em: 06 de abril de 2021. Disponível em: https://www.paho.org/pt/covid19.

REDDY, V. P.et al. Pharmacokinetics under the COVID-19 storm. Authorea Preprints, 2020.

SOBRINHO, N. P.; CAMPOS, J. F.; SILVA, R. C. Eventos adversos a medicamentos relacionados às potenciais interações medicamentosas graves em pacientes com doenças cardiovasculares. Revista Gaúcha de Enfermagem, v. 41, 2020.

YUNES, L. P.; COELHO, T. A.; ALMEIDA, S. M. Principais interações medicamentosas em pacientes da UTI-adulto de um hospital privado de Minas Gerais. Revista Brasileira de Farmácia Hospitalar e Serviços de Saúde, v. 2, n. 3, 2011.

ZHANG, L.; LIU, Y. Potential interventions for novel coronavirus in China: A systematic review. Journal of medical virology, v. 92, n. 5, p. 479-490, 2020. 